\title{
Reduced PLP1 expression in induced pluripotent stem cells derived from a Pelizaeus-Merzbacher disease patient with a partial PLP1 duplication
}

\author{
Keiko Shimojima ${ }^{1,2}$, Takahito Inoue ${ }^{3}$, Yuki Imai ${ }^{4}$, Yasuhiro Arai $^{5}$, Yuta Komoike ${ }^{6}$, Midori Sugawara $^{2}$, \\ Takako Fujita $^{3}$, Hiroshi Ideguchi ${ }^{3}$, Sawa Yasumoto ${ }^{3}$, Hitoshi Kanno ${ }^{7}$, Shinichi Hirose ${ }^{3}$ and \\ Toshiyuki Yamamoto ${ }^{2}$
}

Pelizaeus-Merzbacher disease (PMD) is an X-linked recessive disorder characterized by dysmyelination of the central nervous system (CNS). We identified a rare partial duplication of the proteolipid protein 1 gene (PLP1) in a patient with PMD. To assess the underlying effect of this duplication, we examined PLP1 expression in induced pluripotent stem (iPS) cells generated from the patient's fibroblasts. Disease-specific iPS cells were generated from skin fibroblasts obtained from the indicated PMD patient and two other PMD patients having a 637-kb chromosomal duplication including entire PLP1 and a novel missense mutation (W212C) of PLP1, by transfections of OCT3/4, C-MYC, KLF4 and SOX2 using retro-virus vectors. PLP1 expressions in the generated iPS cells were examined by northern blot analysis. Although PLP1 expression was confirmed in iPS cells generated from two patients with the entire PLP1 duplication and the missense mutation of PLP1, iPS cells generated from the patient with the partial PLP1 duplication manifesting a milder form of PMD showed null expression. This indicated that the underlying effect of the partial PLP1 duplication identified in this study was different from other PLP1 alterations including a typical duplication and a missense mutation.

Journal of Human Genetics (2012) 57, 580-586; doi:10.1038/jhg.2012.71; published online 14 June 2012

Keywords: Pelizaeus-Merzbacher disease (PMD); proteolipid protein 1 gene (PLP1); induced pluripotent stem (iPS) cells; partial duplication; dysmyelination

\section{INTRODUCTION}

Pelizaeus-Merzbacher disease (PMD; MIM \#312080) is an X-linked recessive neurodegenerative disorder characterized by dysmyelination of the central nervous system (CNS). Patients with PMD often present with nystagmus as the initial symptom, and psychomotor developmental delay associated with spasticity and ataxia is seen later in development. ${ }^{1-3}$ The proteolipid protein 1 gene (PLP1; MIM \#300401), located on chromosome $\mathrm{Xq} 22.2$, is the gene responsible for PMD. It encodes 2 isoforms, PLP1 and DM20, as a consequence of differential splicing of exon 3. The genetic basis of PMD is unique because twothirds of PLP1 abnormalities identified in PMD patients are duplications of small chromosomal segments that include PLP1. The remaining one-third of $P L P 1$ abnormalities are nucleotide alterations in the PLP1 coding sequence. The nucleotide alterations in PLP1 are varied and are scattered along the entire coding region of $P L P 1 .{ }^{1-3}$

Because PLP1 is mainly expressed in oligodendrocytes in the CNS and cultured skin fibroblasts express low levels of PLP1, gene expression in the fibroblasts has been analyzed by comparative reverse-transcription (RT)-PCR analysis. ${ }^{4,5}$ The use of technology to establish induced pluripotent stem (iPS) cells has now made it possible to examine gene expression and function in greater detail. ${ }^{6}$ In 2007, Takahashi et al. established iPS cells from human skin fibroblasts. ${ }^{7}$ This revolutionary technology has stimulated and accelerated research in embryogenesis and genetics. In this study, we established iPS cells from skin fibroblasts of patients with PMD and examined PLP1 expression. This is the first report analyzing PLP1 expression in PMD disease-carrying iPS cells.

\section{MATERIALS AND METHODS}

Subjects

For our ongoing study identifying genomic mutations in PLP1, three new patients with dysmyelination were referred to us for genetic diagnosis based on the clinical diagnosis of PMD. ${ }^{8}$ Clinical information and radiographic findings by MRI for the patients were obtained from attending doctors. Based on

${ }^{1}$ Precursory Research for Embryonic Science and Technology (PRESTO), Japan Science and Technology Agency (JST), Kawaguchi, Japan; ${ }^{2}$ Tokyo Women's Medical University Institute for Integrated Medical Sciences, Tokyo, Japan; ${ }^{3}$ Department of Pediatrics, School of Medicine, Fukuoka University, Fukuoka, Japan; ${ }^{4}$ Department of Pediatrics and Child Health, Nihon University School of Medicine, Tokyo, Japan; ${ }^{5}$ Tokyo Metropolitan Tobu Medical Center for Persons with Developmental/Multiple Disabilities, Tokyo, Japan; ${ }^{6}$ Department of Hygiene and Public Health, Tokyo Women's Medical University, Tokyo, Japan and 7 Department of Transfusion Medicine and Cell Processing, Tokyo Women's Medical University, Tokyo, Japan

Correspondence: Dr T Yamamoto, Institute for Integrated Medical Sciences, Tokyo Women's Medical University, Kawada-cho 8-1, Shinjuku-ward, Tokyo 162-8666, Japan. E-mail: yamamoto.toshiyuki@twmu.ac.jp

Received 19 March 2012; revised 15 May 2012; accepted 16 May 2012; published online 14 June 2012 
approval by the ethical committees at the institutions, written informed consent was obtained from each patient and/or their family. Peripheral blood samples were collected from the patients and genotyping was performed as described. ${ }^{8}$ After genetic diagnosis of PMD was made, another written informed consent for the iPS cell study was obtained from each patient and/ or their family. Skin fibroblasts were collected from three patients and a healthy male control.

\section{Genotyping of the patients}

Genomic DNAs were extracted from peripheral blood samples from patients and others by using standard methods. Initial screening for PLP1 duplication was performed by multiplex ligation-dependent probe amplification analysis by using the PLP1 Kit (P022; MRC-Holland, Amsterdam, The Netherlands) according to the manufacturer's instruction. ${ }^{9}$ In case of $P L P 1$ duplication, the aberration region was confirmed by microarray-based comparative genomic hybridization $(\mathrm{aCGH})$ using the Agilent Human 105A CGH Kit (Agilent Technologies, Santa Clara, CA, USA) as described previously. ${ }^{8}$ To detect the small duplication in Patient 1, a custom array was designed using e-array, a web-based software (https://earray.chem.agilent.com/earray/), and 29918 probes in chrX:98000000-104500000, around PLP1, were selected. The average interval of the probes was $217 \mathrm{bp}$ in this region.

PLP1 duplication was confirmed by two-color fluorescence in-situ hybridization as described previously. ${ }^{8}$ Two bacterial artificial chromosome clones, RP11-75D20 (located at Xp22.13) and RP11-832L2 (located at Xq22.2), were selected from the UCSC Human Genome Browser (http://genome.ucsc.edu/) and used as probes. The fixed metaphase and interphase spreads of the specimens were derived from patients' peripheral blood samples and generated iPS cells. The direction of the duplicated segment identified in Patient 2 was analyzed by fiber-fluorescence in-situ hybridization analysis as described previously. $^{8}$

PCR and direct sequencing of all seven exons of PLP1 was performed by standard methods using the primers reported by Hobson et al. ${ }^{4}$ The designs of the primers for all exons and the breakpoint searches of the duplicated segments in Patient 1 are listed in Supplementary Table 1.

\section{Cell culture}

Human fibroblasts, the Plat-E Retroviral Packaging Cell Line (Cell Biolabs, San Diego, CA, USA), 293FT cells (Life Technologies, Foster City, CA, USA) and mouse fibroblast STO cell line (SNL) feeder cells (ECACC, Salisbury, UK) were grown in Dulbecco's modified Eagle's medium (DMEM 14247-15; Nacalai Tesque, Japan) containing $10 \%$ fetal bovine serum and $0.5 \%$ penicillin and streptomycin (Life Technologies). Human iPS cells were maintained on SNL feeder cells treated with mitomycin C in Primate ES Cell Culture Medium supplemented with $4 \mathrm{ng} \mathrm{ml}^{-1}$ recombinant basic fibroblast growth factor (\# RCHEMD001; Repro CELL, Yokohama, Japan) and passaged as described previously. ${ }^{710}$

\section{Generation of iPS cells}

Disease-specific iPS cells were generated from patients' skin fibroblasts as previously described. ${ }^{7}$ Briefly, recombinant lentivirus produced from 293FT cells, in which pLenti6/UbC/mSlc7a1 (AddGene, Cambridge, MA, USA) was transfected by use of Virapower Lentiviral Expression System (Life Technologies), was infected into cultured fibroblasts for $24 \mathrm{~h}$. Then, four retroviruses produced with Plat-E Packaging Cells (Cell biolabs), in which pMXs-hOCT3/4, pMXs-hSOX2, pMXs-hKLF4 and pMXs-hc-MYC (AddGene) were transferred independently, were infected into mSlc7al-expressing human fibroblasts. Six days after retroviral infection, the fibroblasts were placed onto mouse fibroblast SNL feeder cells (ECACC, Salisbury, UK) at the appropriate concentration. The following day, DMEM 14247-15 (Nacalai Tesque, Japan) was replaced with Primate ES Cell Culture Medium supplemented with $4 \mathrm{ng} \mathrm{ml}^{-1}$ recombinant basic fibroblast growth factor (\# RCHEMD001; Repro CELL, Yokohama, Japan). Thirty days after transduction, each embryonic stem (ES) cell-like colony was individually placed onto SNL feeder cells. Each colony was tested to determine whether they had indeed acquired pluripotency. After validation, ${ }^{10}$ three independent iPS cell clones were selected from the candidates generated from each patient's skin fibroblasts.

\section{Validation of the pluripotency of iPS cells}

Initially, alkaline phosphatase staining was performed for validation of iPS cells. Leukocyte Alkaline Phosphatase (AP) kit 86R (Sigma-Aldrich, St Louis, MO, USA) was used for this purpose.

Reactivation of endogenous pluripotency genes and the silencing of artificially induced retroviral transgenes indicated successful reprogramming of putative iPS cell clones. To confirm this, RT-PCR analysis and real-time PCR were performed as described below.

Total RNAs were extracted from iPS cells using ISOGEN (Nippon Gene, Tokyo, Japan) and contaminating genomic DNAs were removed by DNase (Takara, Ohtsu, Japan) according to the manufacturer's instructions. Subsequently, total RNAs were reverse transcribed into complementary DNAs by using the Superscript VILO cDNA Synthesis Kit (Life Technologies) according to the manufacturer's instructions.

Quantitative real-time PCR was performed for OCT3/4, SOX2, KLF4, $C-M Y C, N A N O G, R E X 1$, GAPDH and actin beta using the Power SYBR Green PCR Master Mix (Life Technologies) and analyzed with the 7300 RealTime PCR System (Life Technologies). Primer sequences are shown in Supplementary Table 1.

Immunocytochemistry was also performed for all putative iPS cells. For this purpose, the following primary antibodies were used: anti-SSEA4 (1:200, MAB1435, R\&D systems, Minneapolis, MN, USA), anti-OCT3/4 (1:200, AF1759, R\&D systems), anti-TRA-1-60 (1:200, MAB4360, Millipore, Billerica, MA, USA), and Anti-TRA-1-81 (1:200, MAB4381, Millipore). Secondary antibodies included Alexa488-conjugated donkey anti-mouse IgG, Alexa488conjugated goat anti-mouse IgM, and Alexa594-conjugated donkey antimouse IgG (1:1000, Life Technologies). Nuclei were stained with Hoechst 33342 (1:1000, Life Technologies).

\section{Validation of the differentiation ability of iPS cells}

Determination of the differentiation ability of established iPS cells is important for the selection of putative iPS cell clones. To confirm their pluripotency to differentiate into three embryonic germ layers, we used floating cultivation to form embryoid bodies as described previously. ${ }^{10}$ iPS cells were grown as floating cultures for 8 days. After embryoid body formation, the cells were cultured on gelatin-coated dishes for an additional 8 days.

Immunocytochemistry was performed to confirm expression of the three germ layers as described elsewhere. ${ }^{10}$ In this case, three primary antibodies were used; anti- $\beta$ III tubulin (1:1000, MRB435P, Covance, Princeton, NJ, USA) as the ectoderm marker, anti- $\alpha$ smooth muscle actin (1:200, A2547, SigmaAldrich) for mesoderm, and anti- $\alpha$ AFP (1:100, A8452, Sigma-Aldrich) for endoderm. Donkey anti-mouse IgG labeled with Alexa Fluor 594 and donkey anti-rabbit IgG labeled with Alexa Fluor 488 (1:1000, Life Technologies) were used as secondary antibodies. Nuclei were stained with Hoechst 33342 (1:1000, Life Technologies) for nuclear staining.

\section{Validation of the karyotypes of iPS cells}

To check the artificial chromosomal rearrangements, conventional G-banding by trypsin treatment stained with Giemsa and aCGH analyses using the same methods described above were performed for the generated iPS cell clones. iPS cell lines that acquired chromosomal rearrangements were eliminated from this study.

\section{Database analysis}

Preliminary gene expression analysis was performed using online data sets. Two microarray data sets, GSM242095 for adult human dermal fibroblasts and GSM241846 for iPS cells (clone 201B7), ${ }^{7}$ were retrieved from NCBI Gene Expression Omnibus (GEO) and analyzed using GeneSpring GX10 (Agilent Technologies).

\section{Northern blotting}

The full-length mRNA of PLP1 $(920 \mathrm{bp})$ and a partial sequence of actin beta (ACTNB; MIM \#102630) mRNA (91 bp) were amplified by RT-PCR by using Human Brain Total RNA (\#636530, Clonetech, Mountain View, CA, USA) as a template. Primer sequences are listed in Supplementary Table 1. The PCR product was subcloned into pGEM-T Vector System (Promega, Madison, WI, 
USA) and grown in LB Broth overnight. Plasmid DNAs were extracted by an automated DNA isolation system, PI-80X (Kurabo, Osaka, Japan). DNA inserts were digested with SacI and SacII restriction enzymes. Following agarose gel electrophoresis, product bands were excised and extracted using the QIAquick Gel Extraction Kit (QIAGEN, Hilden, Germany). The DNA fragments were then labeled using $\left[\alpha^{32} \mathrm{P}\right]$ dCTP (PerkinElmer, Waltham, MA, USA) and used as probes for northern blotting.

Hybridization was performed as described previously. ${ }^{11}$ Briefly, $30 \mu \mathrm{g}$ of total RNA was extracted using ISOGEN (Nippon Gene, Tokyo, Japan) according to the manufacturer's instructions, separated on a $1 \%$ agarose $/ 0.6 \mathrm{~m}$ formaldehyde gel, visualized using Radiant Red RNA Stain (Bio-Rad, Hercules, CA, USA), transferred to a nylon membrane and subsequently hybridized for $24 \mathrm{~h}$ with either PLP1 or ACTNB probes. Images were captured using the FLA-5100 scanner (Fujifilm, Tokyo, Japan).

Initial analysis included seven samples: mitomycin-treated and -untreated SNL feeder cells, Epstein-Barr virus-infected immortalized lymphocytes derived from a normal human control, human skin fibroblasts derived from the normal control, iPS cells generated from the normal human control and two brain samples purchased from a provider (Human Fetal Brain Total RNA \#636526 and Human Brain Total RNA \#636530, Clonetech). Subsequent analysis included the 12 iPS cell lines generated in this study.

\section{RESULTS}

\section{Clinical features}

Patient 1 was a 16-year-old male, born by spontaneous delivery at 40 weeks gestation, with a weight of $3054 \mathrm{~g}$. Soon after birth he showed nystagmus. At 4 months, he exhibited poor neck control and was diagnosed with spastic paraplegia. Psychomotor development was moderately delayed with walking alone at his age of 2 years and his intelligence quotient was estimated below 50 . At 15 years, he was prescribed medication for depression. At that time, his fine motor ability allowed the use of chopsticks but he needed a wheel chair to move. His speech was dysarthric. One month later, he had an epileptic attack and was admitted to the hospital. An electroencephalogram revealed occipital spikes. Although auditory brain response was normal, brain magnetic resonance imaging (MRI) revealed a pattern of mild dysmyelination (Figures $1 \mathrm{a}$ and $\mathrm{b}$ ).

Patient 2 was a 46-year-old male with two healthy female siblings. As he lacked neck control at 1 year of age, he was diagnosed with spastic cerebral palsy. Then, at 4 years, he could turn over but could not sit unaided. He lacked the ability to speak effectively, being limited to two-word sentences. At 15 years, he could use a wheel chair by himself. Subsequently, the quality of his daily life declined gradually. At 39 years, MRI revealed atrophic white matter displaying dysmyelination (Figure 1c). At present, he can move only his upper body very slowly and is bedridden. He is able to comprehend what his siblings say, but he is severely dysarthric and is able to speak only a few words very slowly.

Patient 3 was a 32-month-old boy with a birth weight of $3869 \mathrm{~g}$ delivered at 39 weeks gestation. He has a healthy brother. Owing to respiratory problems since birth, he was intubated and tracheostomy was performed at 58 days. He also required tube feeding. $\mathrm{He}$ is currently bedridden and has continuous nystagmus. Auditory-brainresponse audiometry showed no waves after the first wave. A brain MRI revealed high-intensity lesions of the white matter in a T2weighted image, indicating severe hypomyelination (Figure 1d).

\footnotetext{
Molecular analyses

Initial multiplex ligation-dependent probe amplification analysis using a PLP1 Kit (P022; MRC-Holland) identified duplications of PLP1 in Patient 1 and 2 (data not shown). ${ }^{9}$ Patient 2 had a duplication of all 7 exons of PLP1, and subsequent aCGH analysis
}
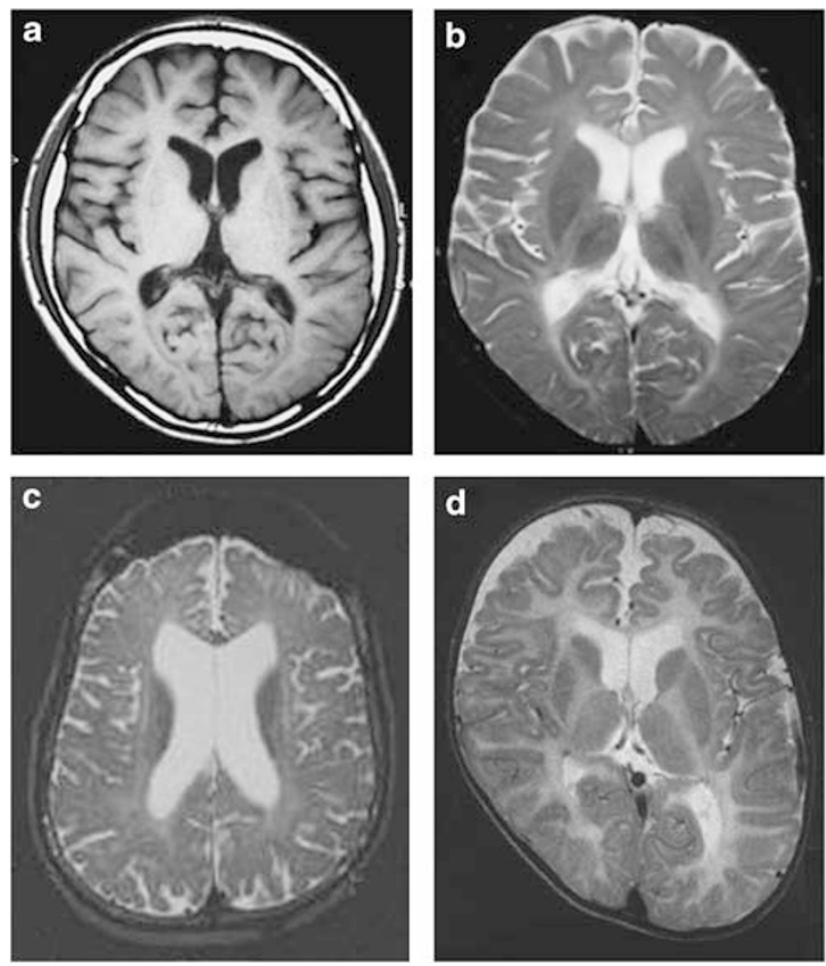

Figure 1 Brain MRI findings of the patients T1- (a) and T2 (b) weighted images of Patient 1 show mild and diffuse volume loss of the brain and high-intensity signals of the deep white matter in T2 indicating mild dysmyelination. T2-weighted image of Patient 2 (c) shows diffuse volume loss resulting in the dilatation of the ventricles and dysmyelination in the white matter. T2-weighted image of Patient 3 (d) shows extremely hypomyelinated pattern with high intensity in all white matter.

by using the Human Genome CGH Microarray $105 \mathrm{~K}$ (Agilent Technologies) revealed that the duplicated region was chrX:102519000-103155851 (636851 bp) with an average $\log _{2}$ ratio of +0.83 , which is a typical duplication region seen in PMD patients with PLP1 duplications (Figure 2a). The duplication was confirmed by fluorescence in-situ hybridization (Figure 2b), and the direction of the duplicated segment, including PLP1, was shown to be in a tandem configuration by fiber-fluorescence in-situ hybridization analysis (Figure 2c).

The duplication identified in Patient 1 was unique because only the first 3 exons (exons 1-3) of PLP1 were included in the duplicated region. To confirm this partial duplication, we designed a custom aCGH chip and used it to detect the precise duplication region. As shown in Figure 3a, the duplicated region was chrX:102 912 361-102 928360 (15999bp) with an average $\log _{2}$ ratio of +0.72 . To determine the location of the duplicated segment, we sought to detect the breakpoint by PCR direct sequencing, using primers $\mathrm{A}$ and B (Supplementary Table 1). A 775-bp band was obtained and re-sequenced (Figures $3 \mathrm{~b}$ and c). Ultimately, an extremely small duplication of $16208 \mathrm{bp}$, which has never been previously reported, was identified. The sample from Patient 1's mother was also analyzed and she was found to be a carrier of this duplication (Figures $3 \mathrm{~b}$ and $\mathrm{c}$ ).

In Patient 3, a novel missense mutation, c.636G > C (W212C), was identified in exon 5 of PLP1 (Figure 2d). The PLP1 sequence is completely conserved among species and this novel mutation was not identified in 100 normal control samples (50 males and 50 females). This patient's mother declined to have her genotype analyzed. 
a
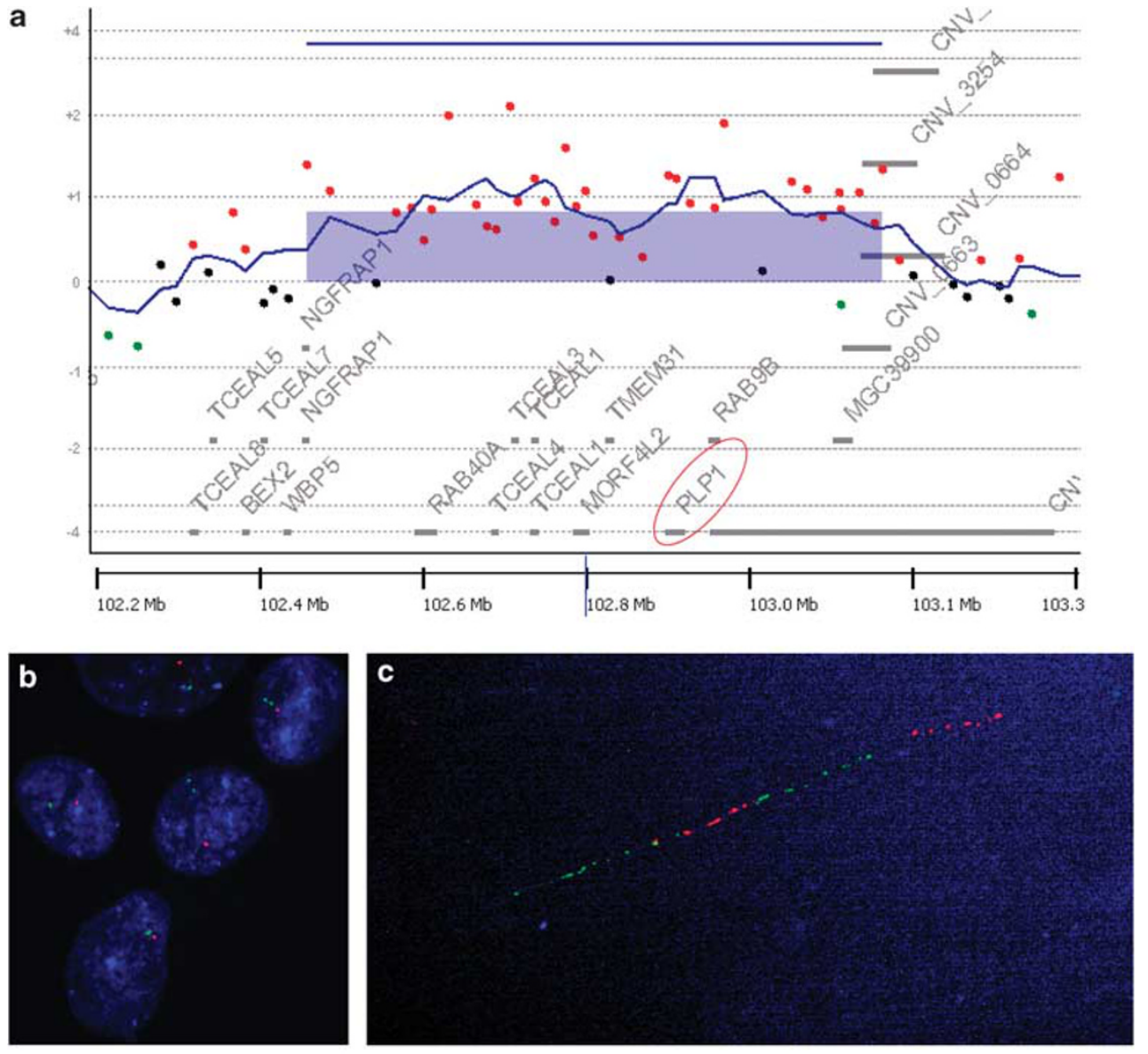

d c.636

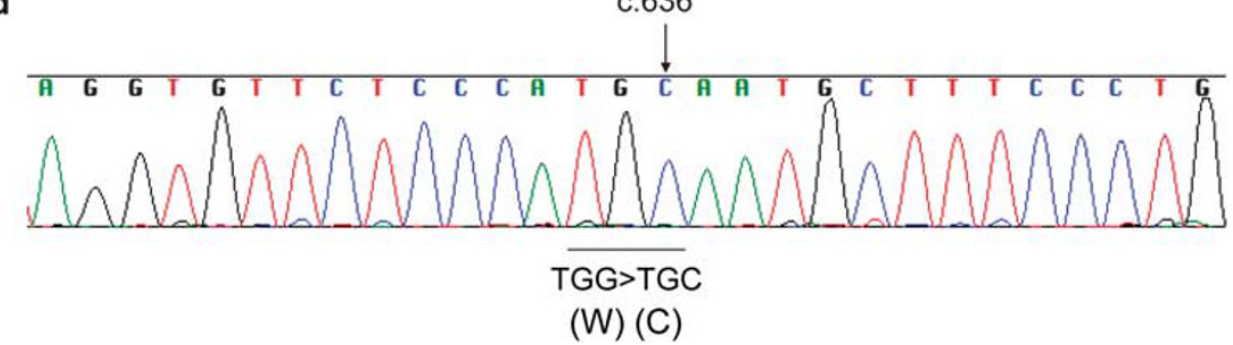

Figure 2 Genotyping and cytogenetic analyses for Patients 2 and 3. (a) A microchromosomal duplication including PLP1 is shown in GeneView of Agilent Genomic Workbench (Agilent Technologies). The location of $P L P 1$ is highlighted by a red circle. (b) Interphase fluorescence in-situ hybridization analysis shows two green signals labeled on RP11-832L2 (located at Xq22.2) in the nucleus. Red signals labeled on RP11-75D20 (located at Xp22.13) are the marker for $\mathrm{X}$ chromosome. (c) Fiber-fluorescence in-situ hybridization analysis indicates tandem configuration of the duplicated segments labeled with green and red probes. (d) Electropherogram shows a novel missense mutation c.636G >C (W212C) in Patient 3.

\section{Generation of iPS cells}

We successfully generated iPS cells from three patients with PMD and a normal male control (Supplementary Figure 1). At least three independent clones were validated using the following three categories: (1) silencing of four transfected genes (OCT3/4, C-MYC, KLF4 and SOX2; Supplementary Figure 2); (2) expression of endogenous pluripotency genes (OCT3/4, SOX2, KLF4, C-MYC, NANOG and REX1; Supplementary Figures 3 and 5); and (3) confirmation of the differentiation potency by immunocytochemistry (Supplementary Figure 4). Karyotype and aCGH analyses for the resulting iPS cells showed no artificial chromosomal rearrangements.

\section{PLP1 expression}

Preliminary PLP1 expression levels were compared between two online data sets for human skin fibroblasts and iPS cells. The results showed that PLP1 expression levels were $\times 40.70\left(\log _{2}=6.38\right)$ higher in iPS cells than in skin fibroblasts (Supplementary Figure 6). Subsequently, our initial experiments for PLP1 expression in several samples were performed by northern blot analysis, which revealed predominant PLP1 expression in the brain (fetal brain had weaker expression than adult brain). Although the other samples showed no PLP1 expression, we could detect the PLP1 band in iPS cells (Figure 4); the differentiation between two isoforms for PLP1 and DM20 could not be detected owing to small size differences as same as 


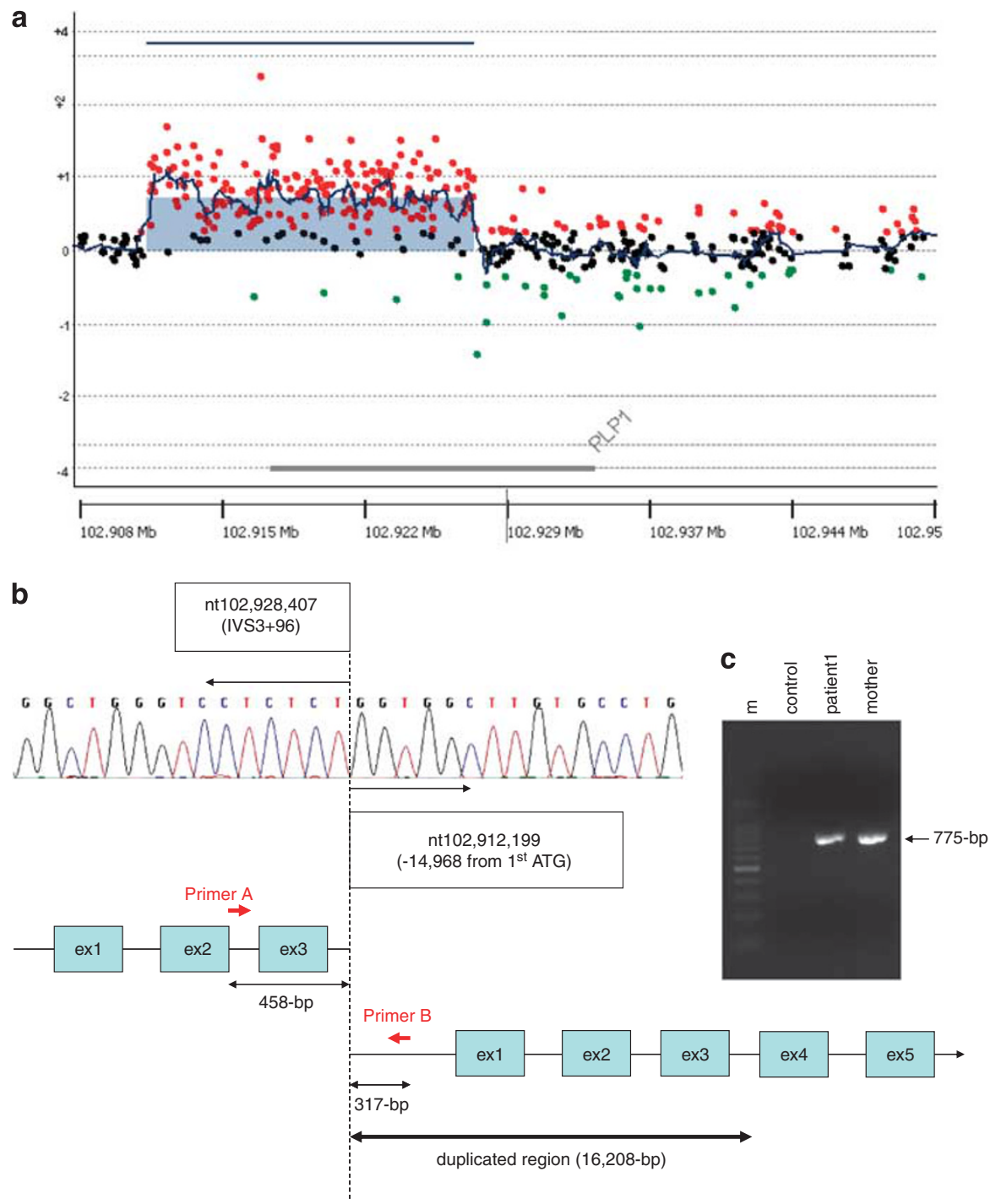

Figure 3 Molecular analyses of the PLP1 duplication identified in Patient 1. (a) The result of a custom-designed aCGH shows partial duplication of $P L P 1$. (b) A schematic representation of the breakpoint analyzed by PCR and subsequent sequencing. (c) A 775 bp PCR product including a breakpoint is amplified by Primer A and B and is shown in electrophoresis. m; molecular marker OneSTEP Ladder 50 (Nippon Gene).

the previous study. ${ }^{12}$ Finally, total RNA samples extracted from the 12 iPS cell clones generated in this study were analyzed. Although we could detect the PLP1 band in iPS cells from normal individual and Patients 2 and 3, we could not detect the PLP1 band in the iPS cells generated from Patient 1, indicating null expression of PLP1 caused by the partial duplication of PLP1 (Figure 5). In Patients 2 and 3, PLP1 signals appeared to be somewhat stronger than in controls, but because of the large variation in signal intensity among different cell lines, it was inconclusive in our limited experiments.

\section{DISCUSSION}

In this study, we identified different PLP1 abnormalities in three patients with PMD. Patient 3 showed a novel missense mutation, c.636G $>$ C (Tyr212Cys), which is in the extramembrane region of the PLP1 protein. ${ }^{2}$ A missense substitution in the same codon, but resulting in a change into a different amino acid, c.634T $>C$ (Tyr212Arg), has been reported to be a pathogenetic mutation by others. ${ }^{13}$ Frequently, a cysteine residue changes the three-dimensional protein conformation drastically owing to disulfide bond formation with other cysteines. ${ }^{14}$ Thus, the amino-acid substitution to cysteine in our patient is likely a pathogenetic mutation, causing PMD. Previous genotype-phenotype correlation study showed that the phenotype of patients with PLP1 missense mutations was more severe than those with $P L P 1$ duplications. ${ }^{1-3}$ Indeed, Patient 3 showed severely delayed psychomotor development complicated by respiratory and feeding difficulties. His condition can be classified as form 0 according to the classification proposed by Cailloux et al., ${ }^{13}$ as form 0 is the most severe form of PMD. Dysmyelination in this patient was particularly severe.

Patient 2 had a $0.6-\mathrm{Mb}$ duplication including PLP1. This size is typical for PMD patients with PLP1 duplications. ${ }^{8,15,16}$ This patient is now 43 years old and does not show any deterioration of neurological abilities. Despite being bedridden, he can verbally communicate with several words. His clinical condition can be classified as form 2, because his maximum motor ability was sitting. His dysmyelination is milder than that of Patient 3.

The most intriguing result in this study is the partial duplication of PLP1 identified in Patient 1. Although there have been reports 


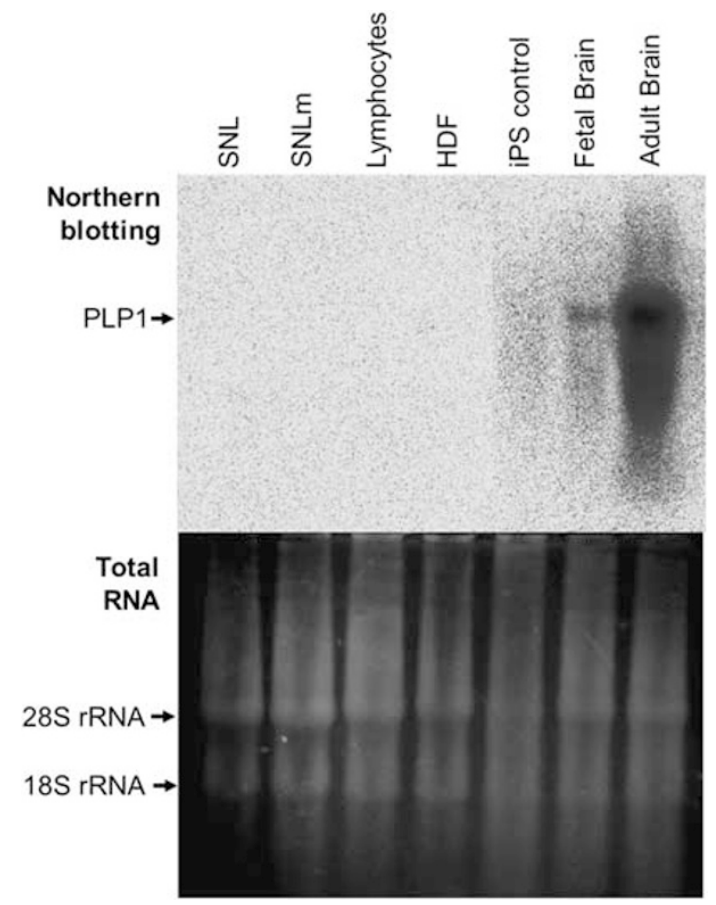

Figure $4 P L P 1$ expression analysis by the northern blotting for various samples. (Upper) Predominant expression of PLP1 is shown in the brain samples. Although control iPS cells show weak expression, there are no expressions of $P L P 1$ in the other samples. (Bottom) Agarose gel staining for the total RNAs before subsequent northern blotting indicates the same amounts of total RNAs loaded in each lane. SNLm, SNL-feader cells treated with mitomycin; HDF, human dermal fibroblasts

of partial deletions in PLP1 identified by multiplex ligation-dependent probe amplification analysis, ${ }^{9}$ this is the first report of a partial duplication of PLP1. The duplicated segment included the promoter region and the first three exons. Therefore, we hypothesized that a very short mRNA or long fusion mRNA might be expressed by this duplication together with the normal mRNA. To confirm this hypothesis, we analyzed PLP1 expression by using northern blot analysis, the only way to detect the length and the quantity of mRNAs. As expression levels of PLP1 in skin fibroblasts were too low to be examined by northern blot analysis, we generated iPS cells from the patients. Contrary to expectation, northern blot analysis showed no PLP1 bands in the iPS cell generated from the fibroblasts of Patient 1. Although there may be a limitation to detect short-unstable mRNAs in our method, this possibly indicated that the expression of PLP1 mRNA was disturbed by the PLP1 partial duplication identified in Patient 1. Regarding the clinical severities of the patients, Patient 1 showed milder phenotype than Patient 2, and his condition can be classified as form 3. Previous genotype-phenotype correlation study have shown that patients with PLP1 missense mutations show severe manifestation associated with severe hypomyelination, which is recognized as the consequence of accumulated mutant protein in the endoplasmic reticulum as a gain-of-toxic function of the mutant protein. $^{2}$ Excessive PLP1 protein resulting from genomic duplications may accumulate in late endosomes/lysosomes, promoting its incorporation into other myelin components. ${ }^{1}$ In contrast, patients with PLP1 null mutations escape severe impairments because of the absence of any gain-of-toxic function. ${ }^{2,13,17}$ Indeed, knockout mice with a functionally null $P l p 1$ gene do not develop classical signs of Plp1-related disease; their

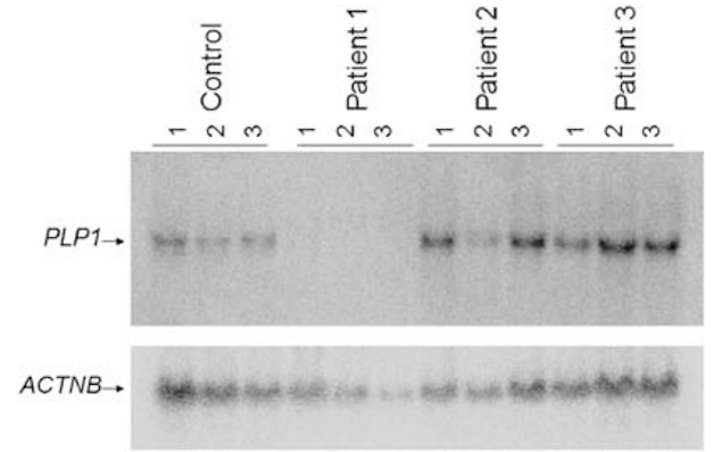

Figure $5 P L P 1$ expression analyses using northern blotting. Three iPS cells of Patient 1 show no PLP1 band, whereas the other iPS cells from normal control, Patient 2 and Patient 3 show expressions of PLP1. ACTNB (actin beta) is used for internal control.

oligodendrocytes develop normally and synthesize compact myelin sheaths. ${ }^{3,18}$ However, the mice show ultrastructural abnormalities, including swelling of the small-diameter axons and late-onset axonal degeneration. ${ }^{3,19}$ Consequently, a loss-of-function mutation of PLP1 does not induce oligodendrocyte cell death, possibly serving as a mechanism underlying the milder phenotypic consequences observed in patients with null PLP1 mutations. Although length-dependent axonal degeneration has been described in PLP1 null mutations, ${ }^{20}$ there is no information about peripheral neuropathy in Patient 1. Thus, it was unclear whether the clinical condition of Patient 1 is compatible with that of PLP1 null mutations. However, the lack of PLP1 expression in iPS cells derived from Patient 1 clearly demonstrated that the underlying mechanism of PMD in Patient 1, with a partial PLP1 duplication, is different from the other two patients in this study.

Immortalized lymphocytes and skin fibroblasts derived from patients are often used for expression studies or biological analyses, as these cells are easy to be obtained and handled. However, many tissue-specific genes are not sufficiently expressed by these cells; PLP1 being one of them. Although there are reports examining PLP1 expression by RT-PCR, using mRNA extracted from skin fibroblasts, ${ }^{4,5,21}$ the expression of PLP1 mRNA in skin fibroblasts is too low to be examined by northern blotting as shown here. In this study, our microarray database search showed over 40 times higher PLP1 expression in iPS cells than that in skin fibroblasts. Our initial northern blot analysis confirmed faint but detectable PLP1 expression in iPS cells, whereas no expression was observed in skin fibroblasts, lymphocytes or SNL feeder cells. This study also confirmed a lack of PLP1 expression in SNL feeder cells. Therefore, this study demonstrates that iPS cells express endogenous PLP1, and that the possibility of contamination from SNL feeder cells or original skin fibroblasts can be excluded. Although being detectable by northern blotting, PLP1 expression in iPS cells appeared to be much lower than that in mature oligodendrocytes and may be simply cryptic rather than functional. If so, this allows us to evaluate the native transcriptional level of each mutant (and wild-type) PLP1 allele, which is the primary focus of our study. Meanwhile, terminal differentiation of iPS cells into the oligodendrocyte lineage would result in an enhanced PLP1 expression with functional consequence. However, this requires technological breakthrough in the induction of terminal differentiation into oligodendrocyte lineage, which is currently unavailable. 
In conclusion, we identified the first PMD patient having a partial PLP1 duplication. The absence of PLP1 expression in iPS cells, generated from the patient's skin fibroblasts, proved the underlying effects of the partial PLP1 duplication for the PMD development.

\section{CONFLICT OF INTEREST}

The authors declare no conflict of interest.

\section{ACKNOWLEDGEMENTS}

We thank the patients and their families for their cooperation. This work was mainly supported by JST PRESTO program (KS) and was partially supported by Grant-in-Aid for Scientific Research on Innovated Areas 'Foundation of Synapse and Neurocircuit Pathology' and Grant-in-Aid for scientific research from Health Labor Sciences Research Grants from the Ministry of Health, Labor and Welfare, Japan (TY). This work was supported in part by Grant-inAid for Scientific Research (A) 21249062, Japan Society for the Promotion of Science (JSPS) and 'High-Tech Research Center' Project for Private Universities: matching fund subsidy from the Ministry of Education, Culture, Sports, Science and Technology, 2006-2010 'The Research Center for the Molecular Pathomechanisms of Epilepsy, Fukuoka University', Research Grants (21B-5) for Nervous and Mental Disorder from the Ministry of Health, Labor and Welfare and Health and Labor Science Research Grant 21210301, KB220001 from the Ministry of Health, Labor and Welfare, and Adaptable and Seamless Technology Transfer Program through Target-driven R\&D (A-STEP) Exploratory Research, Japan Science and Technology Agency (JSP) (SH). KS thanks Hayashi Memorial Foundation for Female Natural Scientists for grant aid support.

WEB SITE: NCBI GEO; http://www.ncbi.nlm.nih.gov/guide/genes-expression/ OMIM; http://omim.org/

1 Inoue, K. PLP1-related inherited dysmyelinating disorders: Pelizaeus-Merzbacher disease and spastic paraplegia type 2. Neurogenetics 6, 1-16 (2005).

2 Garbern, J.Y. Pelizaeus-Merzbacher disease: Genetic and cellular pathogenesis. Cell. Mol. Life Sci. 64, 50-65 (2007).

3 Woodward, K.J. The molecular and cellular defects underlying Pelizaeus-Merzbacher disease. Expert. Rev. Mol. Med. 10, e14 (2008).

4 Hobson, G.M., Huang, Z., Sperle, K., Sistermans, E., Rogan, P.K., Garbern, J.Y. et al. Splice-site contribution in alternative splicing of PLP1 and DM20: molecular studies in oligodendrocytes. Hum. Mutat. 27, 69-77 (2006).

5 Regis, S., Grossi, S., Corsolini, F., Biancheri, R. \& Filocamo, M. PLP1 gene duplication causes overexpression and alteration of the PLP/DM20 splicing balance in fibroblasts from Pelizaeus-Merzbacher disease patients. Biochim. Biophys. Acta. 1792, 548-554 (2009)
6 Inoue, H. Neurodegenerative disease-specific induced pluripotent stem cell research. Exp. Cell. Res. 316, 2560-2564 (2010).

7 Takahashi, K., Tanabe, K., Ohnuki, M., Narita, M., Ichisaka, T., Tomoda, K et al. Induction of pluripotent stem cells from adult human fibroblasts by defined factors. Cell 131, 861-872 (2007).

8 Shimojima, K., Inoue, T., Hoshino, A., Kakiuchi, S., Watanabe, Y., Sasaki, M. et al. Comprehensive genetic analyses of PLP1 in patients with Pelizaeus-Merzbacher disease applied by array-CGH and fiber-FISH analyses identified new mutations and variable sizes of duplications. Brain Dev. 32, 171-179 (2010).

9 Warshawsky, I., Chernova, O.B., Hubner, C.A., Stindl, R., Henneke, M., Gal, A. et al. Multiplex ligation-dependent probe amplification for rapid detection of proteolipid protein 1 gene duplications and deletions in affected males and carrier females with Pelizaeus-Merzbacher disease. Clin. Chem. 52, 1267-1275 (2006).

10 Ohnuki, M., Takahashi, K. \& Yamanaka, S. Generation and characterization of human induced pluripotent stem cells. Curr. Protoc. Stem Cell Biol. Chapter 4, Unit 4A2 (2009).

11 Yamamoto, T., Feng, J.H., Higaki, K., Taniguchi, M., Nanba, E., Ninomiya, H. et al. Increased NPC1 mRNA in skin fibroblasts from Niemann-Pick disease type $\mathrm{C}$ patients. Brain Dev. 26, 245-250 (2004).

12 Iwaki, A., Muramoto, T., Iwaki, I., Furumi, H., Dario-deLeon, M.L., Tateishi, J. et al. A missense mutation in the proteolipid protein gene responsible for Pelizaeus-Merzbacher disease in a Japanese family. Hum. Mol. Genet. 2, 19-22 (1993).

13 Cailloux, F., Gauthier-Barichard, F., Mimault, C., Isabelle, V., Courtois, V., Giraud, G. et al. Genotype-phenotype correlation in inherited brain myelination defects due to proteolipid protein gene mutations. Clinical European Network on Brain Dysmyelinating Disease. Eur. J. Hum. Genet. 8, 837-845 (2000).

14 Dhaunchak, A.S. \& Nave, K.A. A common mechanism of PLP/DM20 misfolding causes cysteine-mediated endoplasmic reticulum retention in oligodendrocytes and Pelizaeus-Merzbacher disease. Proc. Natl Acad. Sci. USA 104, 17813-17818 (2007).

15 Lee, J.A., Inoue, K., Cheung, S.W., Shaw, C.A., Stankiewicz, P. \& Lupski, J.R. Role of genomic architecture in PLP1 duplication causing Pelizaeus-Merzbacher disease. Hum. Mol. Genet. 15, 2250-2265 (2006).

16 Woodward, K.J., Cundall, M., Sperle, K., Sistermans, E.A., Ross, M., Howell, G. et al. Heterogeneous duplications in patients with Pelizaeus-Merzbacher disease suggest a mechanism of coupled homologous and nonhomologous recombination. Am. J. Hum. Genet. 77, 966-987 (2005).

17 Sistermans, E.A., de Wijs, I.J., de Coo, R.F., Smit, L.M., Menko, F.H. \& van Oost, B.A. A (G-to-A) mutation in the initiation codon of the proteolipid protein gene causing a relatively mild form of Pelizaeus-Merzbacher disease in a Dutch family. Hum. Genet. 97, 337-339 (1996).

18 Klugmann, M., Schwab, M.H., Puhlhofer, A., Schneider, A., Zimmermann, F., Griffiths, I.R. et al. Assembly of CNS myelin in the absence of proteolipid protein. Neuron 18, 59-70 (1997).

19 Griffiths, L. Klugmann, M., Anderson, T., Yool, D., Thomson, C. Schwab, M.H. et al. Axonal swellings and degeneration in mice lacking the major proteolipid of myelin. Science 280, 1610-1613 (1998).

20 Garbern, J.Y., Yool, D.A., Moore, G.J., Wilds, I.B., Faulk, M.W., Klugmann, M. et al. Patients lacking the major CNS myelin protein, proteolipid protein 1, develop lengthdependent axonal degeneration in the absence of demyelination and inflammation. Brain 125, 551-561 (2002)

21 Mikesova, E., Barankova, L., Sakmaryova, I., Tatarkova, I. \& Seeman, P. Quantitative multiplex real-time PCR for detection of PLP1 gene duplications in PelizaeusMerzbacher patients. Genet. Test. 10, 215-220 (2006).

Supplementary Information accompanies the paper on Journal of Human Genetics website (http://www.nature.com/jhg) 